\title{
Chemical Derivatization of Catecholamines for Gas Chromatography-Mass Spectrometry
}

\author{
Sun-Young Park, Bo-Xin Kang, Quing-Li, Hoon Sik Kim, ${ }^{\dagger}$ Jungae Lee, ${ }^{\ddagger}$ and Jongki Hong* \\ College of Pharmacy, Kyung Hee University, Seoul 130-701, Korea. *E-mail: jhong@khu.ac.kr \\ ${ }^{\dagger}$ Department of Chemistry, Kyung Hee University, Seoul 130-701, Korea \\ ${ }^{\ddagger}$ Korea Institute of Science \& Technology, Seoul 136-791, Korea \\ Received March 22, 2009, Accepted May 11, 2009
}

\begin{abstract}
GC/MS analysis of catecholamines (CAs) in biological sample may produce poor reproducible quantitaion when chemical derivatization is used as the technique to form a volatile derivative. Significant quantities of the side products can be formed from CAs with primary amine during the derivatization reaction under un-optimized conditions. We have tested various chemical derivatization techniques in an attempt to find an optimum derivatization method that will reduce side product formation, enable to separate several catecholamine derivatives in GC chromatogram, and obtain significant improvement of detection sensitivity in GC/MS analysis. Whereas several derivatization techniques such as trimethylsilylation (TMS), trifluoroacylation (TFA), and two step derivatization methods were active, selective derivatization to form O-TMS, $N$-heptafluorobutylacyl (HFBA) derivative using $N$-methyl- $N$-(trimethylsilyl)-trifluoroacetamide (MSTFA) and $N$-methyl-bis(heptafluorobutyramide) (MBHFBA) reagents was found to be the most effective method. Moreover, this derivative formed by selective derivatization could provide sufficient sensitivity and peak separation as well as produce higher mass ion as base peak to use selected ion in SIM mode. Calibration curves based on the use of an isotopically labeled internal standard show good linearity over the range assayed, $1 \sim 5000 \mathrm{ng} / \mathrm{mL}$, with correlation coefficients of $>0.996$. The detection limits of the method ranged from 0.2 to $5.0 \mathrm{ppb}$ for the different CAs studied. The developed method will be applied to the analysis of various CAs in biological sample, combined with appropriate sample pretreatment.
\end{abstract}

Key Words: Catecholamines, Chemical derivatizations, Mass fragmentation patterns, GC/MS analsysis

\section{Introduction}

Quantification of catecholamines (CAs) such as dopamine, epinephrine, and adrenaline etc. in biological sample is requested in the clinical diagnosis of pheochromocytoma and related neurogenic tumors. ${ }^{1,2}$ The profiling analysis of catecholamines is considered a reliable screening test for pheochromocytoma and Parkinson's disease. ${ }^{3-5}$

Quantitation of very low levels of CAs in human urine, blood, and plasma has been an important goal for investigators, and several sensitive techniques have been developed including HPLC with electrochemical (EC) detection, ${ }^{6-8}$ HPLCmass spectrometry (MS) ${ }^{9-11}$ and gas chromatography/MS (GC/ MS) with selected ion monitoring (SIM). ${ }^{12-14}$ Although HPLCEC is a highly sensitive method for the analysis of catecholamines, this method has relatively long analysis time and istroubled by other endogenous compounds. Recently, LC-MS method has been widely used for the analysis of catecholamines in biological fluids. However, LC-MS method has some limitation on the separation, accuracy, and sensitivity for some compounds. In addition, precise quantitation is impaired by the lack of proper internal standards for controlling losses during chemical manipulations. ${ }^{9}$ The GC-MS/SIM method is more universal and provides high sensitivity and high selectivity for many substances, although it often requires chemical derivatization.

GC-MS protocols have been applied to the quantitation of CAs in human urine, blood, and plasma; ${ }^{12-14}$ however, formation of a volatile derivative of CAs is preferentially required.
Various chemical derivatization approaches of catecholamines have been published with current silylation and acylation reagents. All of these methods lead to the formation of trimethylsilyl (TMS) and acylated derivatives. TMS and acylation methods also permit adequate gas chromatographic separation of various CAs, and these derivatives have high GC-MS sensitivity. ${ }^{12}$ Besides these methods, two-step derivatization of catecholamines includes silylation followed by perfluorobenzylation, ${ }^{15}$ methylation, ${ }^{16}$ and acylation. ${ }^{17}$ During chemical derivatization of CAs with primary amine group, significant quantities of side products are also present when reaction conditions are not optimized. The effectiveness of a chemical derivatization procedure depends on main factors including the nature of the derivatizing reagent, the reaction time, and the reaction temperature. Although several derivatization methods have been mainly reported for two or three catecholamines, method for the derivatization of various catecholamines has not been reported in detail.

The aim of this work was to optimize and to compare various derivatization procedures for 8 catecholamines. Six derivatization methods including TMS, TFA reagent and mixture reagents were tested and compared. For optimizing chemical derivatization, the influence of the parameters (reagent amounts, reaction time, and temperature) was tested for each mixture in terms of derivatization yields. We have also studied the linearity on quantitation and limits of detection for the developed derivatization method. This method combined with appropriate sample treatment will be developed a specific screening method, enabling monitoring and measurement of 
catecholamines in human urine, blood, and plasma by GCMS-SIM mode.

\section{Material and Methods}

Chemicals. Authentic catecholamines, dopamine hydrochloride (DA), homovanilic acid (HVA), 3,4-dihydroxy- $L$ phenyl-alanine (DOPA), $D, L$-normetanephrine hydrochloride $(\mathrm{NMN})$ and $( \pm)$-epinephrine hydrochloride (EP), were purchased from Sigma Chemical (St. Louis, MO, USA). 3,4-Dihydroxyphenylacetic acid (DOPAC), 4-hydroxy-3-methoxymadelic acid (VMA), and $D, L$-noradrenaline hydrochloride (NE) were obtained from Fluka (Büchs, Switzerland). The purity of these chemicals is over $97 \%$. Chemical structures of catecholamines studied are depicted in Figure 1.

Derivatization reagents, $N$-methyl- $N$-(trimethylsilyl)-trifluoroacetamide (MSTFA) and $N$-(tert-butyldimethylsilyl)$N$-merhyltrifluoroacetamine (MTBSTFA) were obtained from Sigma-Aldrich Chemical Co. (Milwaukee, WI, USA). N,O-Bis (trimethylsilyltrifluoroacetamide (BSTFA), BSTFA with 1\% TMCS, and $N$-trimethylsilyl-imidazole (TMSI) were obtained Supelco (Bellefonte, PA, USA). N-Methyl-bis(trifluoroacetamide) (MBTFA) and trifluoroacetic anhydride (TFAA) were obtained from Sigma. $N$-Methyl-bis(heptafluorobutyramide) (MBHFBA) was purchased from Macherey-Nagel (Duren, Germany).

Acetonitrile, ethylacetate, methanol, and pyridine were obtained from J. T. Baker (Rockford, IL, USA). Hydrochloric acid was obtained from Merck (Darmstadt, Germany), and phenanthrene- $d_{10}$ used as internal standard was obtained from Supelco.

Stock Solutions. Catcholamines were dissolved in methanol at a concentration of $1 \mathrm{mg} / \mathrm{mL}$, respectively. However, dopa was dissolved in methanol by addition of $50 \mu \mathrm{L} 6 \mathrm{M}-\mathrm{HCl}$, to improve its solubility. All standard solutions are stable for several weeks when kept at $4{ }^{\circ} \mathrm{C}$. The solution was kept in an amber vial to protect the material from photooxidation.

Derivatization. Catecholamine mixture (each $2 \mu \mathrm{g}$ ) with the internal standard $(2 \mu \mathrm{g})$ was dried under nitrogen stream in a silylated reaction vial before derivatization. The vial was dried under vacuum for $5 \mathrm{~min}$ at room temperature. Silylation of CAs was achieved by addition of $70 \mu \mathrm{L}$ of ethylacetate and 10 , 20 , or $30 \mu \mathrm{L}$ of one of the following silylation agents: MSTFA, MTBSTFA, BSTFA, or TMSI. The reaction mixture was purged with argon for $30 \mathrm{~s}$, tightly capped, vortexed, and heated at $80{ }^{\circ} \mathrm{C}$ for $10 \mathrm{~min}$. To the solution, $20 \mu \mathrm{L}$ of one of the following acylation agents: MBHFBA or MBTFA was added and the solution was heated $80{ }^{\circ} \mathrm{C}$ for $10 \mathrm{~min}$. The reaction time course for the derivatization of CAs by various reagents at $80{ }^{\circ} \mathrm{C}$ was performed for $0,5,10,30$, and 60 min using phenathrene- $d_{10}$ as an internal standard. Derivatized samples were injected onto GC/MS.

To optimize the OTMS, NHFBA derivatization of catecholamines, the influence of the reagent amounts, the reaction temperature and time was studied. A mixture of catecholamines dissolved in ethylacetate, each one at $10 \mu \mathrm{g} / \mathrm{mL}$, was derivatized with MSTFA and MBHFBA at three temperatures: 60 , 80 , and $100{ }^{\circ} \mathrm{C}$; six reaction times: $5,10,15,20,30$, and 60

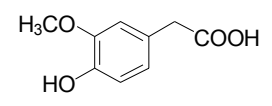

Homovanillic acid (HVA)

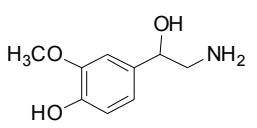

Normetanephrine (NMN)

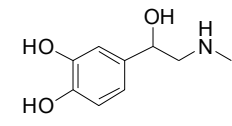

Epinephrine (EP)

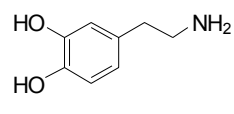

Dopamine (DA)

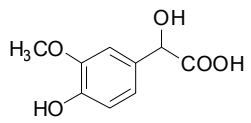

Vanillomandelic acid (VMA)

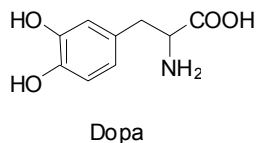

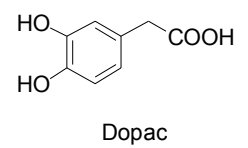

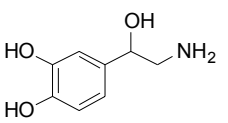

Norepinephrine (NE)
Figure 1. Chemical structures of catecholamines used in this study.

min were tested at each temperature.

GC-MS. An Agilent 5973N (Palo Alto, CA, USA) mass spectrometer (EI mode, $70 \mathrm{eV}$ ) connected to an Agilent 6890 gas chromatograph equipped with a DB-5MS capillary column $(25 \mathrm{~m} \times 0.2 \mathrm{~mm}$ i.d., $0.33 \mu \mathrm{m}$ film thickness, J \& W Scientific, Folsom, CA, USA) was used for analysis. Samples were introduced via split (ratio 10:1) injection with the port at $270{ }^{\circ} \mathrm{C}$. Helium was used as the carrier gas at a flow rate of $1.0 \mathrm{~mL} /$ min. The oven temperature, initially $120{ }^{\circ} \mathrm{C}$ held for $3 \mathrm{~min}$, was ramped to $180^{\circ} \mathrm{C}$ at a rate of $10^{\circ} \mathrm{C} / \mathrm{min}$ and then raised to $190{ }^{\circ} \mathrm{C}$ at a rate of $5^{\circ} \mathrm{C} / \mathrm{min}$, then ramped to $280{ }^{\circ} \mathrm{C}$ at a rate of $15{ }^{\circ} \mathrm{C} / \mathrm{min}$, held for $5 \mathrm{~min}$. The mass spectrometer interface temperature was set at $280{ }^{\circ} \mathrm{C}$. The manifold temperature was maintained at $230{ }^{\circ} \mathrm{C}$. The mass spectrometer was operated with scan mode between 100 and $600 \mathrm{amu}$. For the monitoring and confirmation analysis, the selected ion monitoring (SIM) mode was used. SIM, using their base peak and second abundant ions for derivatized CAs and isotopically labeled internal standards, was used for quantitative analysis. The relative peak area was obtained by dividing the integrated area of base peak by that of internal standard.

Calibration Curve. Calibration curves were constructed for 8 CAs by adding $10 \mathrm{ng}$ of the internal standard to each of five known concentrations. The standards were dried and derivatized with MSTFA/MBHFBA as described above. The linear equations and correlation coefficients $\left(\mathrm{R}^{2}\right)$ were obtained by the least-squares method.

\section{Results and Discussion}

Derivatization and Mass Spectrometry of Catecholamines. Since catecholamines have generally dihydroxyl groups and an amine group, each analyte possesses more than three potential sites of derivatization. To study the influence of derivatization reagents on reaction yield, 8 catecholamines were derivatized with (A) MSTFA, (B) TFAA, (C) MSTFA/MBTFA, (D) MTBSTFA/MBTFA, (E) MSTFA/MBHFBA, and (F) TMSI/MBHFBA and injected into GC/MS. The total ion chromatograms of CAs-derivatives after derivatization with above reagents are displayed in Figure 2. The characteristic ions of various CA-derivatives under EI mode are summarized 
Table 1. Characteristic ions of CA-derivatives using various reagents

\begin{tabular}{|c|c|c|c|c|c|c|c|c|c|c|}
\hline \multirow{2}{*}{ Compound } & \multicolumn{2}{|c|}{ MSTFA } & \multicolumn{2}{|c|}{ TFAA } & \multicolumn{2}{|c|}{ MSTFA, MBTFA } & \multicolumn{2}{|c|}{ MTBSTFA, MBTFA } & \multicolumn{2}{|c|}{ MSTFA/TMSI, MBHFBA } \\
\hline & MW & ions & MW & ions & MW & ions & MW & ions & MW & ions \\
\hline HVA & 326 & 209311 & 374 & - & 326 & 209311 & 410 & 353281 & 326 & 209311 \\
\hline DA & 369 & 102268 & 441 & 328126 & 393 & 267193 & 477 & 193420 & 493 & 267193 \\
\hline Dopac & 384 & 267179 & 456 & - & 384 & 267179 & 510 & 453179 & 384 & 267179 \\
\hline NMN & 399 & 297102 & 471 & 358345 & 423 & 297267 & 507 & 381450 & 523 & 297267 \\
\hline VMA & 414 & 297371 & 486 & - & 414 & 297371 & 540 & 381483 & 414 & 297371 \\
\hline $\mathrm{NE}$ & 457 & 355102 & 553 & 126440 & 481 & 355265 & 607 & 481149 & 581 & 355265 \\
\hline EP & 471 & 116355 & 567 & 410454 & 495 & 355281 & 623 & 480184 & 595 & 355267 \\
\hline Dopa & 485 & 218267 & 581 & 341467 & 509 & 267179 & 635 & 578179 & 609 & 267179 \\
\hline
\end{tabular}

(A) MSTFA

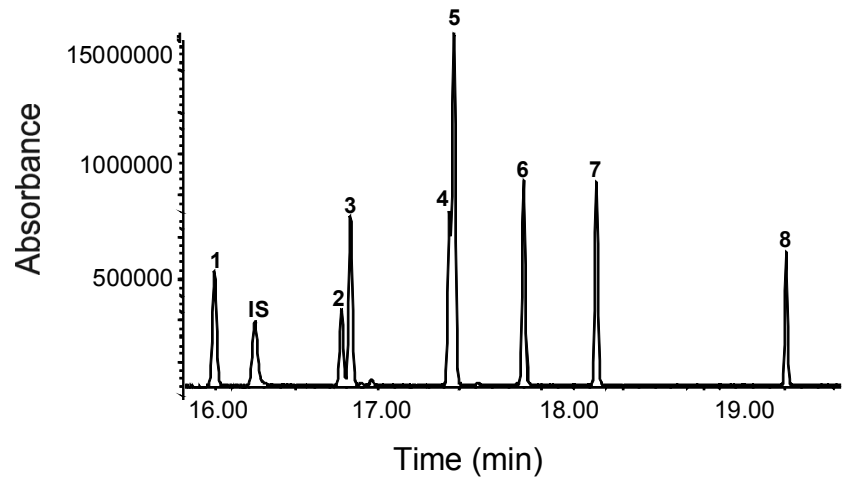

(C) MSTFA/MBTFA

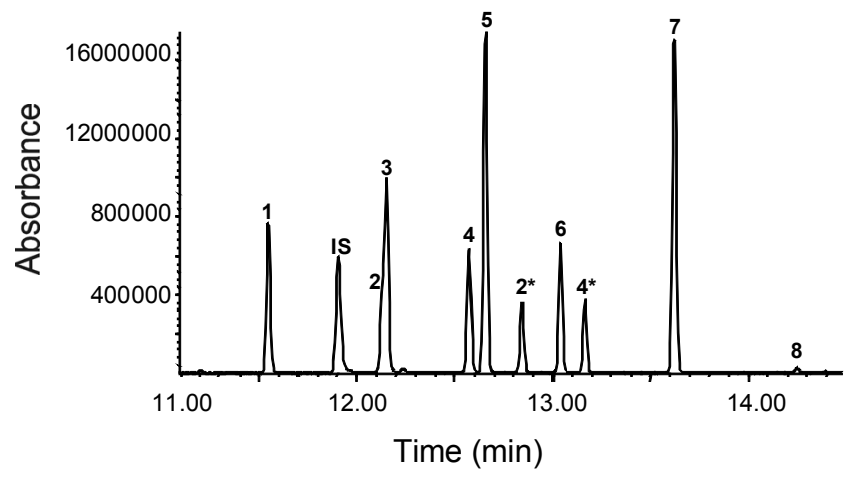

(E) MSTFA/MBTHFBA

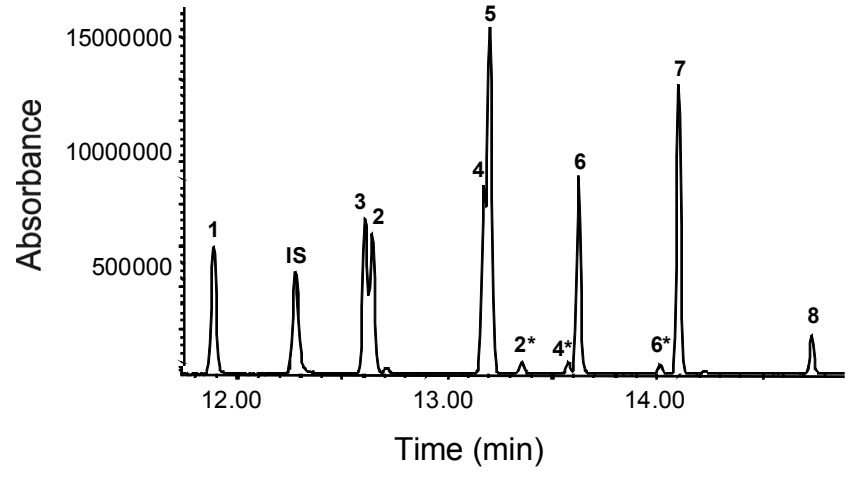

(B) TFAA

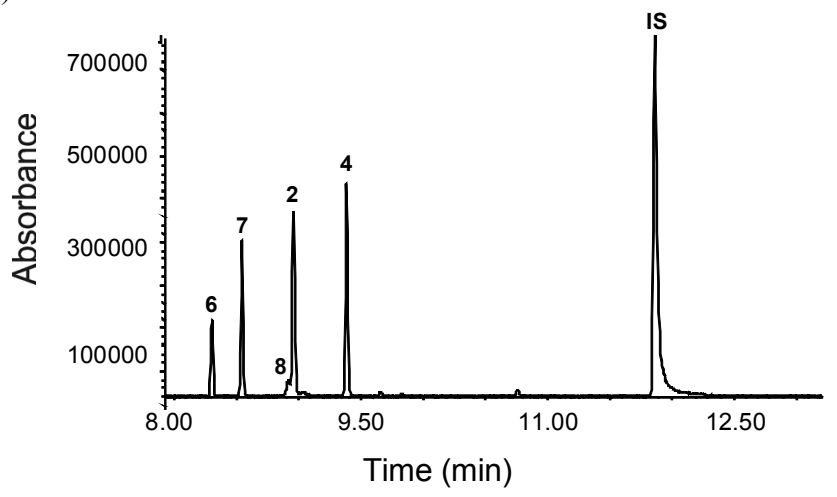

(D) MTBSTFA/MBTFA

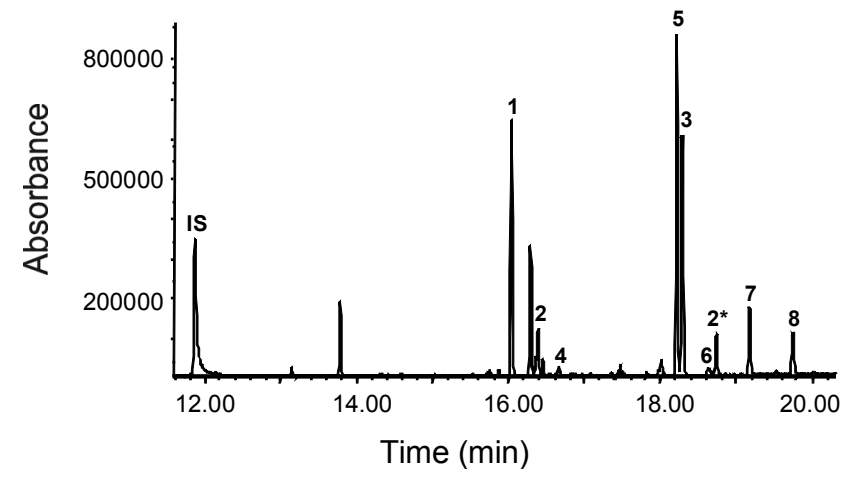

(F) TMS/MBHFBA

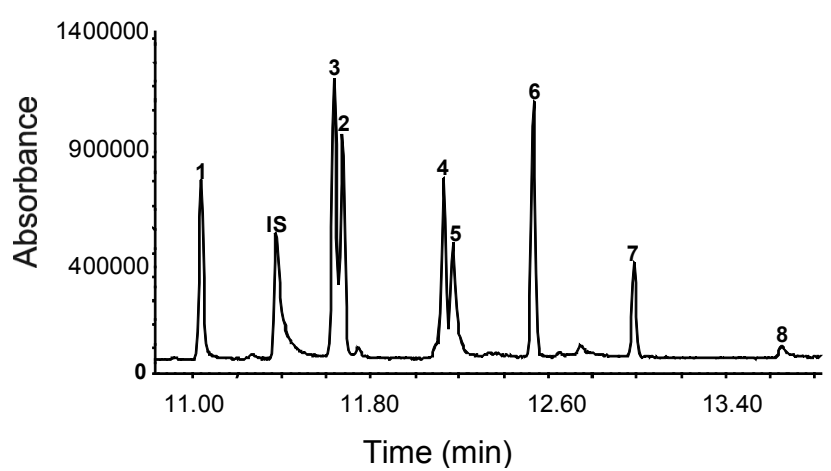

Figure 2. Total ion chromatograms of derivatized catecholamines using (A) MSTFA, (B) TFAA, (C) MSTFA/MBTFA, (D) MTBSTFA/ MBTFA, (E) MSTFA/ MBHFBA, and (F) TMSI/MBHFBA. Peaks identity as follow: 1. HVA; 2. DA; 2*. DA side-product; 3. Dopac; 4. NMN; 4*. NMN side-product; 5. VMA; 6. NE; 6*. NE side-product; 7. EP; and 8. Dopa. The internal standard (IS) was phenanthrene- $d_{10}$. 
(A) TMS

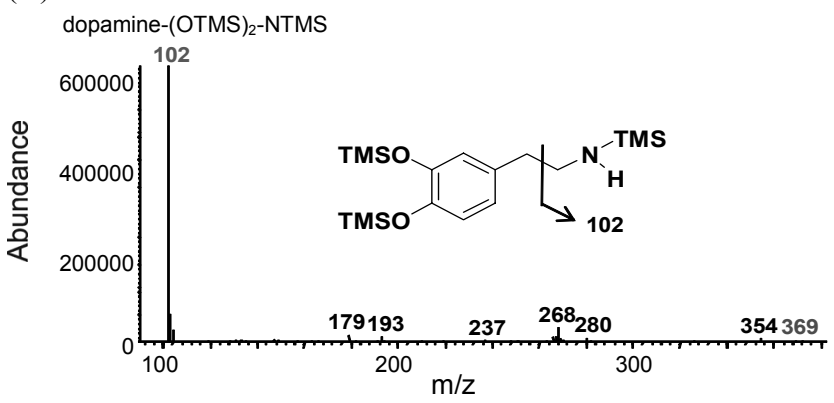

(B) TFA

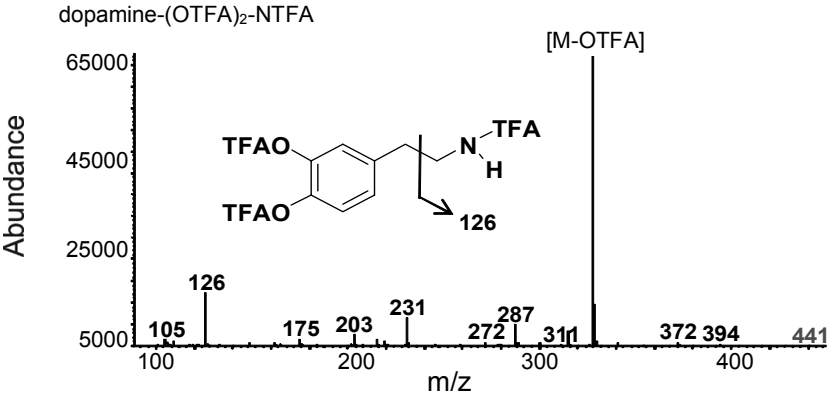

(C) OTMS, NTFA

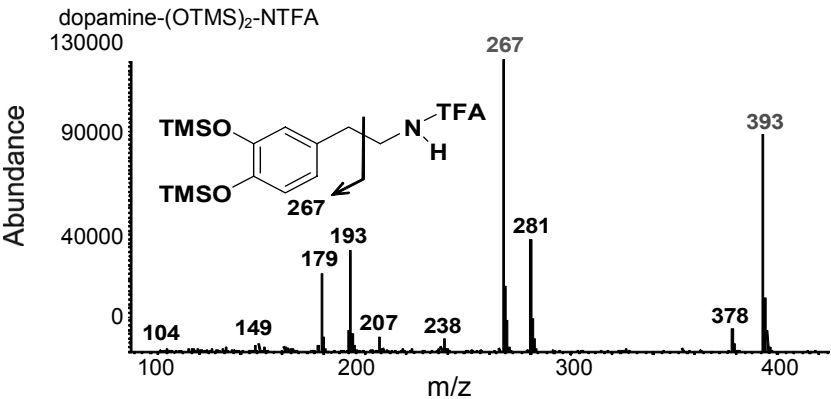

(D) OTBDMS, NTFA

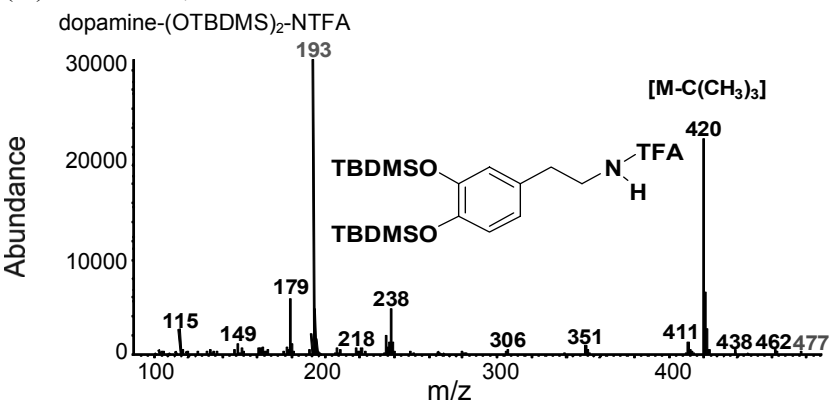

(E) OTMS, NHFBA

dopamine-(OTMS) $)_{2}$-NHFBA

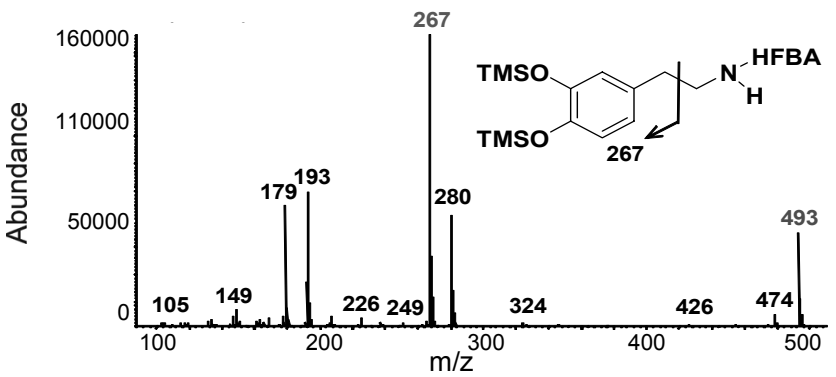

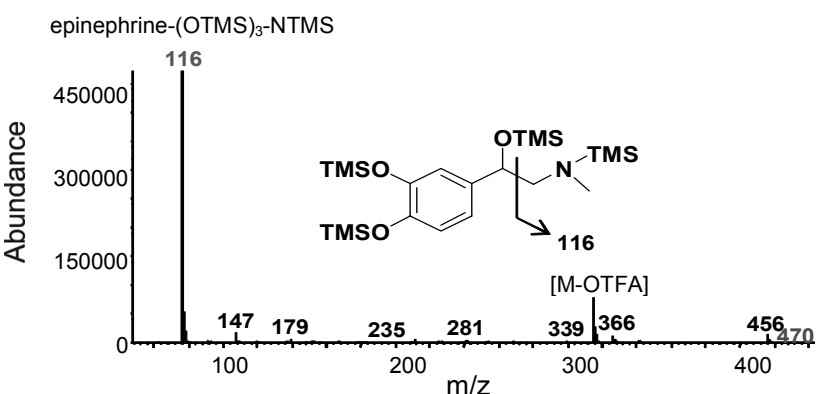
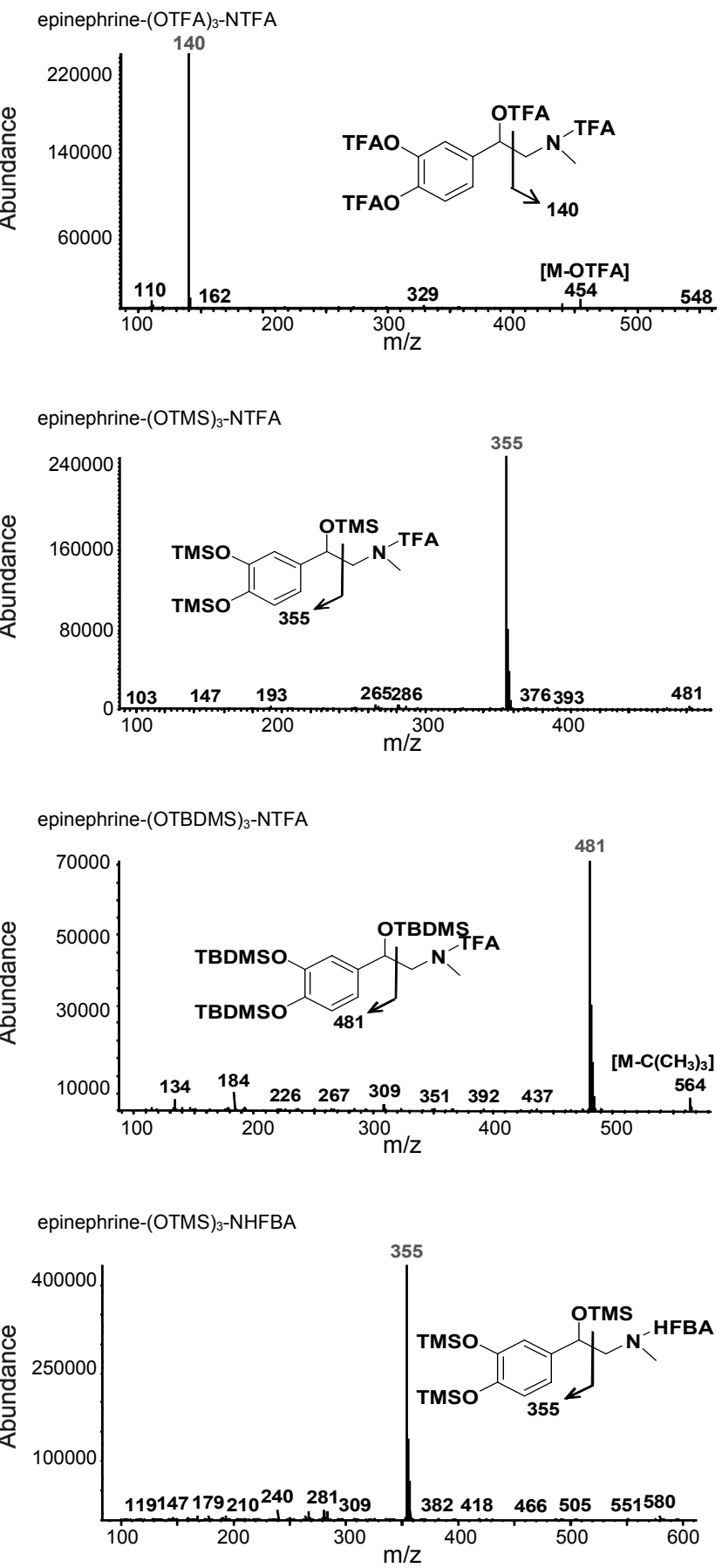

Figure 3. Mass spectra of various dopamine and epinephrine derivatives (A) TMS, (B) TFA, (C) OTMS, NTFA, (D) OBDTMS, NHFBA, and (E) OTMS, NHFBA. 
in Table 1. The base peak and second abundant ion will be used in SIM mode. Molecular ions were generally of low abundance under the given experimental conditions. CA derivatives afforded several characteristic ions due to easy cleavage of benzylic position and the charge initiation was preferentially occurred on the nitrogen atom.

For one step derivatization of CAs with MSTFA, all CA$(\mathrm{OTMS})_{2}$, -NTMS derivatives were shown a reasonable sensitivity and peak resolution. No significant amounts of side products were observed in the chromatogram, as shown in Figure 2-(A). However, most of CA-TMS derivatives produced base peaks at low mass ion under electron ionization mode, as shown in Figure 3. These base peaks at m/z 102 for primary amines or $\mathrm{m} / \mathrm{z} 116$ for secondary amines were formed by $\alpha$-cleavage at charge initiation on nitrogen atom of amine group. These low mass ions could be interfered by matrix components in urine or blood sample during GC/MS-SIM analysis and could lead to inaccurate quantitation and to reduce their detection sensitivity.

As another one step derivatiztaion, TFAA was also applied for the acylation of CAs. After TFA derivatization of CAs with TFAA, reaction mixture should be removed by nitrogen stream for the elimination of trifluoroacetic acid produced from TFAA. As shown in Figure 2-(B), the TFA derivatives of CAs containing amine group were successfully derivatized even though showing relatively low sensitivity. A poor sensitivity of CA-TFA derivatives could be explained probably due to easy loss of trifluoroacetic acid from CA-TFA derivatives. Moreover, acidic CAs such as dopac, homovanilic acid and vanilomandelic acid did not react with TFAA. The mass fragmentation of CA-TFA derivatives in EI-mass spectra led to produce typical characteristic ion $[\mathrm{M}-\mathrm{OTFA}]^{+}$as a base peak.

In the present work, the mixture compounds of CAs were examined after conversion to their corresponding derivatives, prepared respectively by selective O-silylation with the three silylation reagents, followed by selective $\mathrm{N}$-acylation with two acylation reagents. These selective derivatization methods have several advantages compared one step derivatization with MSTFA or TFAA alone. The hydroxyl and carboxylic acid groups react readily with each of the three silylating reagents (MSTFA, BSTFA, and MTBDMSTFA) under mild conditions. The amine groups, on the other hand, were rapidly derivatized with MBTFA or MBHFBA reagent to form amides compounds that are chemically relatively stable and not easy to cleave. Moreover, no acidic byproducts are produced when MBTFA or MBHFBA areused, as are common in derivatization with trifluoroacetic anhydride or heptafluorobutyric anhydride. This eliminated a second evaporation and reconstitution step and extended the useful lifetime of the GC column because acid derivatives were not injected.

The combination of MSTFA/MBTFA reagents has been widely used for selective derivatization of several kinds of drugs and biological active amines. ${ }^{18,19}$ In this study, the derivatization of CAs by two step reaction exhibited a reasonable resolution and sensitivity except for dopa, as shown in Figure 2-(C). The base peaks of OTMS, NTFA (or NHFBA) derivatives are produced at $\mathrm{m} / \mathrm{z} 267$ or 355 by benzylic cleavage to form a stable ion, as shown in Figure 3. The higher mass ions of base peaks in their corresponding EI mass spectra could improve sensitivity and decreased interference effect. However, significant amounts of side products [CA-(OTMS), N(TFA, TMS) ] for CAs with primary amines were produced during derivatization reaction. Furthermore, dopamine and dopac derivatives which produced same base peak at m/z 267 were co-eluted, unable to quantify accurately these compounds.

To reduce the formation of side-product and to separate these compounds, the combination of MTBSTFA/MBTFA reagents was also applied. In general, MTBSTFA has several advantages over MSTFA such as more endurance of hydrolysis and longer retention time in GC analysis to minimize interference effect and enhance resolution. ${ }^{20}$ As shown in Figure 2-(D), overall reaction yield of CAs was shown to be a poor and several side-products were observed. The mass fragmentation patterns of CA-OTBDMS, NTFA derivatives were almost similar to those of CA-OTMS, NTFA derivatives. On the other hand, the elution order of CA-OTBDMS, NTFA derivatives is quite different from those of CA-OTMS, NTFA derivatives. This selective derivatization also produced a significant amount of side products for CAs with primary amine and led to relatively longer retention times of CA-derivatives. The reagent peaks were observed in TIC if not effectively removing of reagent after derivatization.

As another attempt to separate these compounds, the combination of MSTFA/MBHFBA reagents was applied. As can be seen in Figure 2-(E), CA-OTMS, NHFBA derivates were shown to be a reasonable resolution and sensitivity. Under the same chromatographic conditions, the reagent peaks were well separated from those of the CA-derivatives and did not interfere with the detection, in spite of the repeated injection of samples. Therefore, it is not necessary to evaporate the reaction medium. These derivatives also did not show any adsorption effects in the GC system. The mass fragmentation of CA-OTMS, NHFBA derivates are also similar to those of CA-OTMS, NTFA derivates. However, small amounts of side-products [CA-(OTMS) 2 , N(HFBA, TMS)] for CAs with primary amine were also observed when reaction conditions are not optimized.

To protect the formation of side product, the combination of TMSI/MBHFBA was used. TMSI is known to be selectively derivatize only hydroxyl group, not derivatize amine group ${ }^{21}$ due to strong basicity of imidazole. As shown in Figure 2-(F), no significant amounts of side products were observed. However, this derivatization method showed relatively higher baseline and peak tailing than other derivativesin chromatogram because of strong interaction between TMSI and column surface. Thus, repeated injection of this reaction mixture could lead to poor accuracy and short column life time.

The relative response factors (RRFs) of several CAs-chemical derivatives were calculated using the peak area ratio relative to phenanthrene- $d_{10}$ as internal standard, as summarized in Table 2. The RRFs of CA-TFA derivatives were shown relatively low due to their easy hydrolysis. Some of CAs including DA, NMN, NE, and Dopa were very poor sensitivity for OTBDMS, NTFA derivatization. Overall RRFs 
Table 2. Relative response factors of various catecholamine derivatives $(\mathrm{n}=3)$

\begin{tabular}{lccccc}
\hline \multirow{2}{*}{ Compound } & \multicolumn{5}{c}{ Mean \pm S.D. (R.S.D. (\%)) } \\
\cline { 2 - 6 } & OTMS, NTMS & OTFA, NTFA & OTMS, NTFA & OTBDMS, NTFA & OTMS, NHFBA \\
\hline HVA & $1.3 \pm 0.1(4.62)$ & - & $1.0 \pm 0.0(1.99)$ & $1.2 \pm 0.3(25.47)$ & $1.1 \pm 0.1(7.26)$ \\
DA & $0.7 \pm 0.1(8.85)$ & $0.3 \pm 0.1(4.98)$ & $0.6 \pm 0.0(5.09)$ & $0.2 \pm 0.0(16.42)$ & $1.0 \pm 0.1(7.65)$ \\
Dopac & $1.7 \pm 0.1(5.11)$ & - & $1.2 \pm 0.0(2.63)$ & $1.1 \pm 0.0(3.53)$ & $1.3 \pm 0.1(6.37)$ \\
NMN & $1.2 \pm 0.1(5.17)$ & $0.3 \pm 0.1(1.74)$ & $0.7 \pm 0.1(6.98)$ & $0.0 \pm 0.0(16.55)$ & $1.4 \pm 0.0(3.45)$ \\
VMA & $3.4 \pm 0.2(5.32)$ & - & $2.2 \pm 0.1(2.42)$ & $1.6 \pm 0.3(20.94)$ & $2.6 \pm 0.1(4.10)$ \\
NE & $1.7 \pm 0.1(4.91)$ & $0.1 \pm 0.0(21.66)$ & $0.8 \pm 0.1(7.40)$ & $0.1 \pm 0.0(44.55)$ & $1.3 \pm 0.1(4.24)$ \\
EP & $1.7 \pm 0.1(3.90)$ & $0.2 \pm 0.0(21.31)$ & $2.2 \pm 0.1(4.02)$ & $0.3 \pm 0.1(27.65)$ & $2.0 \pm 0.1(5.50)$ \\
Dopa & $0.9 \pm 0.1(7.87)$ & $0.0 \pm 0.0(24.69)$ & $0.0 \pm 0.0(5.35)$ & $0.2 \pm 0.0(26.38)$ & $0.3 \pm 0.0(9.77)$ \\
\hline
\end{tabular}

(A) Amount of reagent
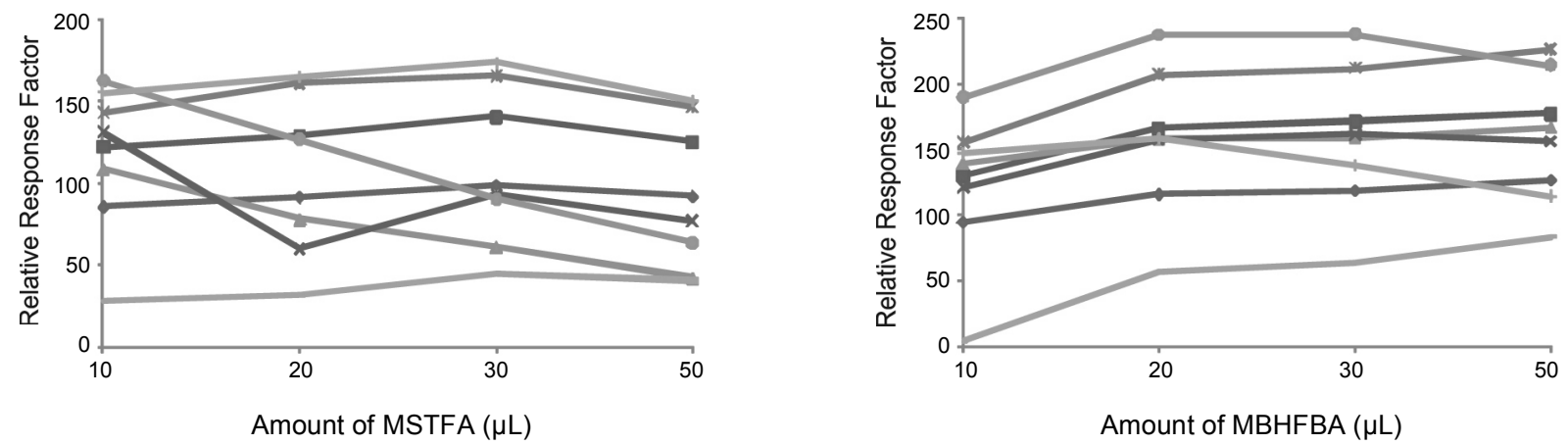

(B) Reaction temperature
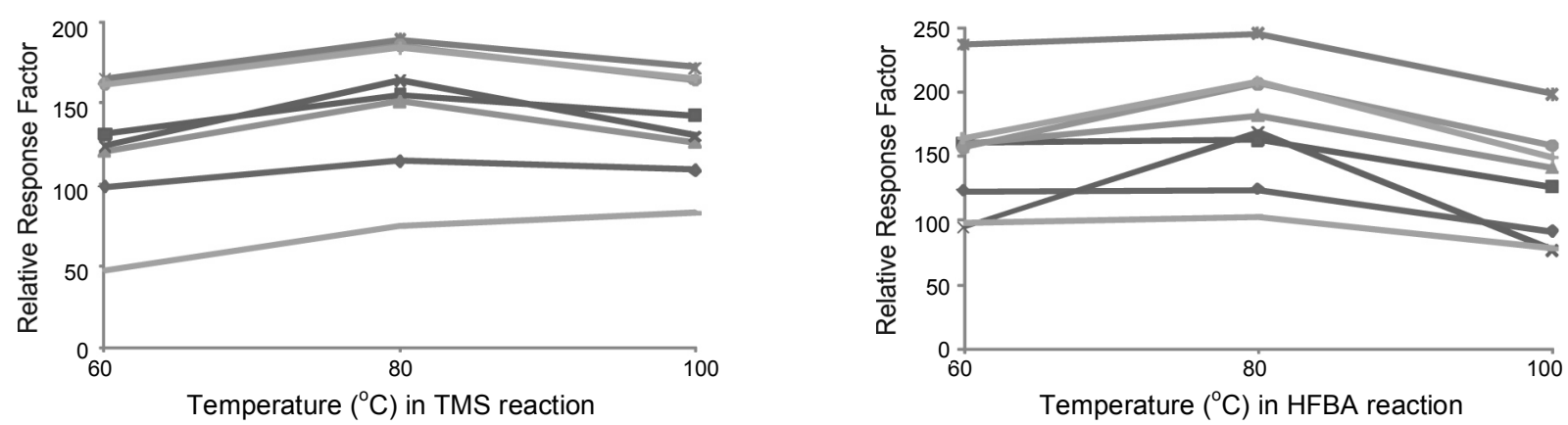

(C) Reaction time
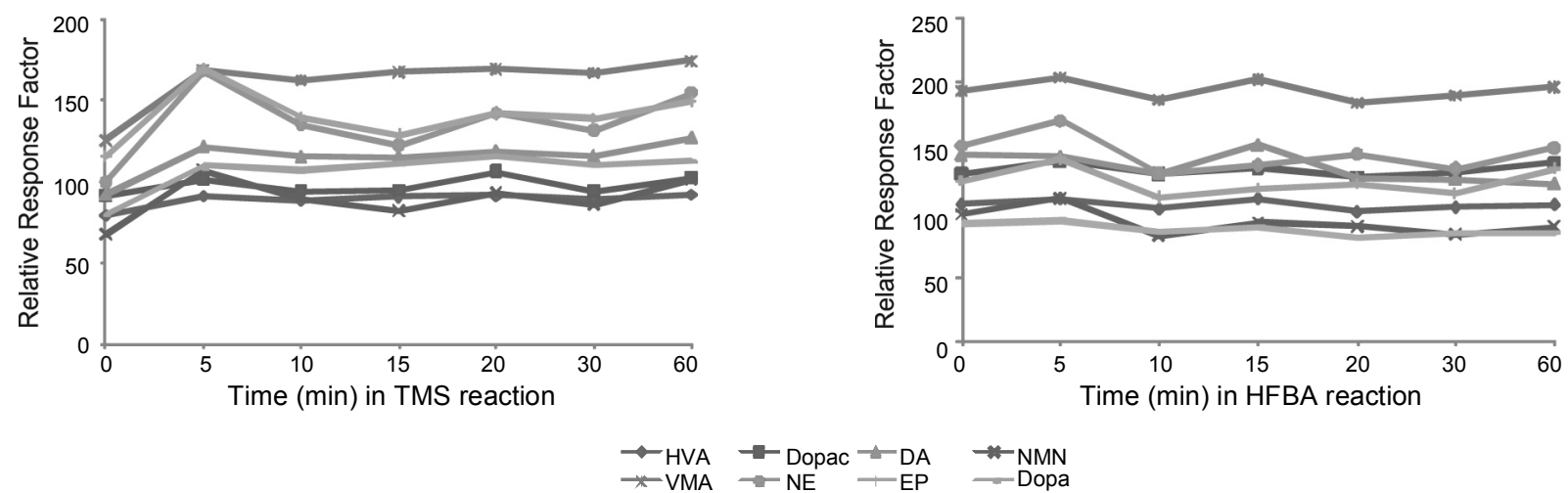

Figure 4. Profiling of CAs-OTMS, NHFBA derivatization reaction according to amount of reagent (A), temperature (B), and time (C). 


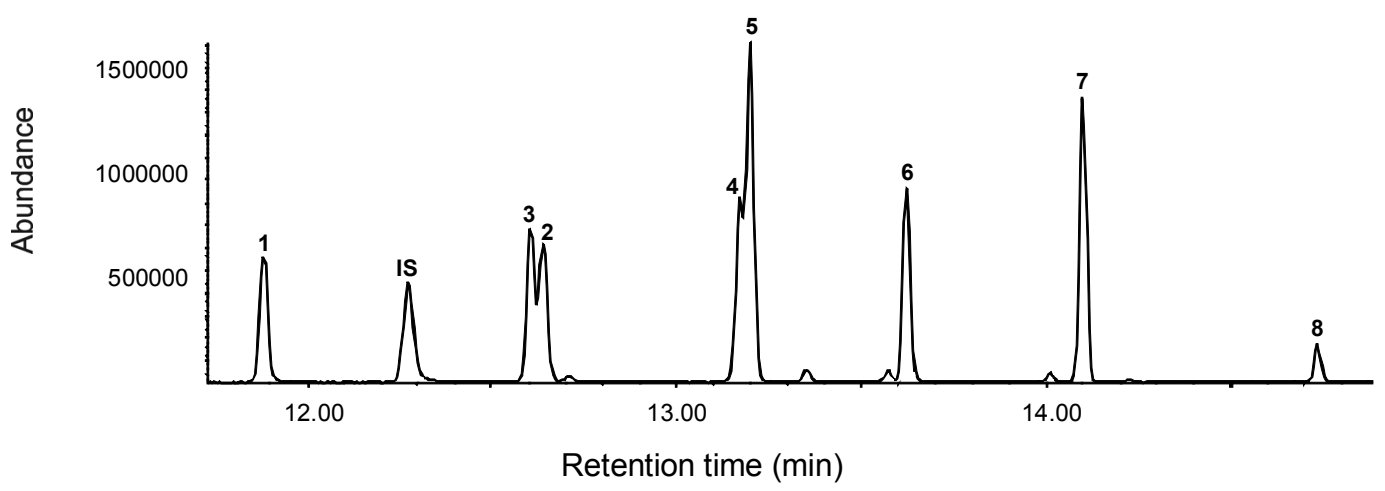

Figure 5. Total ion chromatogram of trimethylsilylated and heptafluorobutylated catecholamines. Peak's identity as follow; 1 . HVA-(OTMS $)_{2}$; 2. DA-(OTMS $)_{2}$, NHFBA; 3. Dopac-(OTMS $)_{3} ; 4$. NMN-(OTMS) $)_{2}$, NHFBA; 5. VMA-(OTMS $)_{3}$; 6. NE-(OTMS) $)_{3}$, NHFBA; 7. EP-(OTMS) 3 , NHFBA; and 8 . Dopa-(OTMS $)_{3}$, NHFBA. The internal standard (IS) was phenanthrene- $d_{10}$.

Table 3. Calibration curve data, correlation coefficients, and limits of detections

\begin{tabular}{|c|c|c|c|c|c|}
\hline \multirow{2}{*}{ Compounds } & \multirow{2}{*}{$\begin{array}{l}\text { Linear range } \\
\quad(\mathrm{ng} / \mathrm{mL})\end{array}$} & \multicolumn{2}{|c|}{ Linear equation } & \multirow{2}{*}{$\begin{array}{l}\text { Correlation } \\
\text { coefficient }\end{array}$} & \multirow{2}{*}{$\begin{array}{c}\text { LOD } \\
(\mathrm{ng} / \mathrm{mL})\end{array}$} \\
\hline & & Slope & Intercept & & \\
\hline HVA-(OTMS $)_{2}$ & $1-5000$ & 0.341 & -4.414 & 0.999 & 0.2 \\
\hline DA-(OTMS $)_{2}$, NHFBA & $10-5000$ & 0.598 & -7.142 & 0.999 & 2.5 \\
\hline Dopac-(OTMS $)_{3}$ & $5-5000$ & 0.630 & -38.91 & 0.999 & 0.5 \\
\hline NMN-(OTMS) $)_{2}$ NHFBA & $5-5000$ & 1.050 & -102.2 & 0.997 & 1.5 \\
\hline VMA-(OTMS $)_{3}$ & $1-5000$ & 1.381 & -43.27 & 0.999 & 0.2 \\
\hline NE-(OTMS) $)_{3}$, NHFBA & $20-5000$ & 1.413 & -97.01 & 0.999 & 5.0 \\
\hline EP-(OTMS) $)_{3}, \mathrm{NHFBA}$ & $20-5000$ & 1.216 & -125.9 & 0.998 & 5.0 \\
\hline Dopa-(OTMS) $)_{3}$, NHFBA & $5-5000$ & 0.361 & -25.92 & 0.999 & 1.0 \\
\hline
\end{tabular}

of the TMS, [OTMS, NTFA], and [OTMS, NHFBA] derivatives were shown similar response, except for Dopa. However, for TMS derivatization, low mass ion as base peak could be interfered with matrix extracted from biological sample. However, the selective derivatization of OTMS, NTFA has a serious problem, producing significant abundance of side products for CAs with primary amine. Although the selective derivatization of OTMS, NHFBA produced side products for CA with primary amine, this method provided a reasonable sensitivity and peak resolution. For DA which is known as one of important markers, OTMS, NHFBA derivatization was shown as the highest response. The CA-OTMS, NHFBA derivatives was not only able to increase the analyte detectability, but also decrease the uncertainty of quantification, producing high mass ion as base peak. Considering of the sensitivity and peak resolution, therefore, the MSTFA/HBHFBA derivatization of several methods was selected and investigated to reduce the formation of side product.

Optimzing the Selective Derivatization of CA-OTMS, NHFBA. To optimize the derivatization of CA-OTMS, NHFBA, the amounts of derivatizing reagents, reaction temperature, and reaction time were tested (Figure 4). Variation of the amount of MSTFA for TMS derivatization, the TMS reaction yield of CAs was very slightly increased as amount of MSTFA increases except for dopamine and norepinephrine. The amounts of dopamine and norepinephrine-TMS derivatives greatly decreased as amount of MSTFA increases, due to formation of side product. It is interesting observation that the TMS/HFBA reaction yield of CAs with primary amine group slightly decreased as amount of MBHFBA increase. Thus, use of excess silylating agents should be avoided. In this study, the amounts of MSTFA and MBHFBA were selected 10 and $20 \mu \mathrm{L}$, respectively.

The reaction temperature and reaction time can strongly affect the kinetic of derivatization reaction. Several derivatization reactions have been performed at high temperature and longer reaction time to increase the reaction rate and yield. However, the amount of side product for CAs with primary amine significantly increased as the temperature and time increase. Though not shown here, the amount of side products could be effectively reduced applying on-column reaction, but the overall reaction yield was much lower than those applying temperature at $80^{\circ} \mathrm{C}$ for $5 \mathrm{~min}$. Thus, if the reaction temperature is set to a high value, a short reaction time is sufficient to achieve a good reaction yield.

Under these conditions, the sufficient reaction yields of CAs were obtained and the formation of side products for CAs with primary amine greatly suppressed within $3 \%$ compared to major products, as shown in Figure 5. Moreover, the overall precision of peak area for these derivatives was shown within $5 \%$, providing satisfactory reproducibility. Although some of CA-derivatives such aspeak number 2 and 3 , and 4 and 5 were partially overlapped in total ion chromatogram, these compounds could be precisely quantified due to observing different 
base peakin SIM chromatogram.

Method Validation. In order to check the linearity of the derivatization method, calibration curves in the concentration range of $1.0 \sim 5000 \mathrm{ng} / \mathrm{mLwere}$ constructed and calculated according to the method of least squares, relating y (the peak area ratio of CA-derivatives to the internal standard) to $\mathrm{x}$ (the concentration of the CAs in $\mathrm{ug} / \mathrm{mL}$ ). Standard mixture solution was analyzed in triplicate. The calibration curves of the analytes showed good linearity within given concentration ranges. Table 3 lists the data for the calibration curves which were linear on the corresponding concentration range, showing above correlation coefficient $\left(\mathrm{R}^{2}\right) 0.996$.

The limit of detection (LOD) was established by considering value 3 times the background noise. In many cases of GC/MS analysis, the LOD can be affected by several factors such as $\mathrm{GC}$ injection volume, injection split ratio, and final solvent volume. The sensitivity of this method could be enhanced by adjusting these factors. The range of LODs achieved from 0.2 to $5 \mathrm{ng} / \mathrm{mL}$ was lower or equivalent to those obtained by other results. ${ }^{12,13}$ It is important to note that clinically significant concentrations of CAs in urine are usually 10 - to 100 -fold higher concentrations than LODs achieved in this study.

\section{Conclusions}

The chemical derivatization method of catecholamines is well established for the sensitive and simultaneous determination the catecholamines by GC/MS. This method is applicable to the analysis of several biological active compounds containing more than one reactive functional group, e.g., phenolalkylamines, $\beta$-blockers, and amino acids. The derivatives are useful for the simultaneous quantification of these compounds in a biological matrix by selected-ion monitoring. Additional application of the method to quantitative measurement of these compounds in biological samples is currently under study in our laboratory.

Acknowledgments. This study was financially supported by the research fund of Kyung Hee University (2008).

\section{References}

1. Eisenhofer, G. Clin. Chem. 2001, 47, 988.

2. Gram, P. E.; Smythe, G. A.; Edwards, G. A.; Lazarus, L. Ann. Clin. Biochem. 1993, 30, 129.

3. Taylor, R. L.; Singh, R. J. Clin. Chem. 2002, 48, 533.

4. Lagerstedt, S. A.; O'Kane, D. J.; Singh, R. L. Clin. Chem. 2004, 50,603 .

5. Andrew, R.; Watson, D. G.; Best, S. A.; Midgley, J. M.; Wenlong, H.; Petty, T. K. H. Neurochem. Res. 1993, 18, 1175.

6. Vuorensola, K.; Siren, H.; Karjalainen, U. J. Chromatogr. B 2003, $788,277$.

7. Monsaingeon, M.; Simonnet, G.; Corcuff, J.-B. Eur. J. Pediatr. 2003, 162, 397.

8. Lionetto, L.; Lostia, A. M.; Stigliano, A.; Cardelli, P.; Simmaco, M. Clin. Chim. Acta 2008, 398, 53

9. De Jong, W. H. A.; Graham, K. S.; Van Der Mollen, J. C.; Links, T. P.; Morris, M. R.; Ross, H. A.; De Vries, E. G. E.; Kema, I. P. Clin. Chem. 2007, 53, 1684.

10. Gu, Q.; Shi, X.; Yin, P.; Gao, P.; Lu, X.; Xu, G. Anal. Chim. Acta 2008, 609, 192.

11. Lagerstedt, S.; O'Kane, D. J.; Singh, R. J. Clin. Chem. 2004, 50, 603.

12. Crockett, D. K.; Frank, E. L.; Roberts, W. L. Clin. Chem. 2002, 48, 332.

13. Bergquist, J.; Sciubisz, A.; Kaczor, A.; Silberring, J. J. Neurosci. Methods 2002, 113, 1.

14. Fauler, G.; Leis, H. J.; Huber, E.; Schellauf, C.; Kerbi, R.; Urban, C.; Gleispach, H. J. Mass Spectrom. 1997, 32, 507.

15. Lhuguenot, J. C.; Maume, B. F. Biomed. Mass Spectrom. 1980, 7,529 .

16. Muskiet, A. J.; Jeuring, H. J.; Nager, G. T.; Druyn, H. W.; Wolthers, B. G. Clin. Chem. 1978, 24, 1899.

17. Kuhlenbeck, D. L.; O’Neill, T. P.; Mack, C. E.; Hoke 1l, S. H.; Wehmeyer, K. R. J. Chromatogr. B 2003, 738, 319.

18. Lho, D. S.; Hong, J. K.; Paek, H. K.; Lee, J. A.; Park, J. J. Anal. Toxicol. 1990, 14, 77.

19. Shin, H.-S.; Donike, M. Anal. Chem. 1996, 68, 3015.

20. Ahn, Y. G.; Kim, H. Y.; Shin, J. H.; Khim, J.; Lee, M. K.; Hong, J. Anal. Chim. Acta 2007, 603, 67.

21. Knapp, D. R. Handbook of Analytical Derivatization Reactions; John Wiley \& Sons: New York, 1979. 